\title{
USO DE TECNOLOGÍA HSPA (HSPA +) Y SU EVOLUCIÓN CON LA GENERACIÓN DE LOS CELULARES.
}

Fausto Orozco, Milton Andrade, Jaime Orozco, Mercedes Villarroel. 


\title{
Uso de tecnología HSPA (HSPA +) y su evolución con la generación de los celulares.
}

\section{Use of HSPA (HSPA +) technology and its evolution to the generation of cell phones}

\author{
Fausto Ernesto Orozco Iguasnia ${ }^{1}$, Milton Temístocles Andrade Salazar ${ }^{1}$, Jaime Benjamín Orozco \\ Iguasnia², Mercedes Villarroel Sánchez²; \\ ${ }^{1}$ Pontificia Universidad Católica del Ecuador Sede Santo Domingo, Ecuador. \\ ${ }^{2}$ Universidad Estatal Península de Santa Elena, Santa Elena, Ecuador. \\ E-mail: oife@pucesd.edu.ec
}

\section{Resumen}

La necesidad la tecnología HSPA+ or Evolved High Speed Packet Access también es conocido como HSPA evolution, surgió con la creciente utilización de los datos y los usuarios que desean velocidades de descarga mucho más eficiente, en Ecuador CNT para el 2014 se propuso implementar 491 radio bases HSPA+ para alcanzar una cobertura del 84 por ciento de la población ecuatoriana y desde entonces ha alcanzado una excelente cobertura a nivel nacional. El objetivo de este trabajo es realizar un análisis de la tecnología HSPA + , así como también su evolución a partir de la generación de los celulares, en la segunda etapa se hablará de HSPA sus ventajas, arquitectura de red, estructura de protocolos en la cual se verá la capa MAC y la capa $R L C$, en la tercera etapa HSDPA su arquitectura de red, sus principales mejoras, estructura de protocolos, handover en HSDPA, en la cuarta etapa HSUPA su arquitectura de red, estructura de protocolos, sus principales mejoras, sus canales y handover HSUPA, en la etapa 5 hablaremos la evolución de HSPA + dentro del release 7 .

Palabras clave: Tecnología HSPA +, MAC, RLC, HSPA, HSDPA, HSUPA, LTE.

\section{Abstract}

The need for HSPA + technology or Evolved High Speed Packet Access is also known as HSPA evolution, emerged with the growing use of data and users wanting download speeds much more efficient, in Ecuador CNT for 2014 set out to implement 491 radio bases HSPA + to reach coverage of 84 percent of the Ecuadorian population and since then reaching an excellent coverage at the national level. The objective of this work is to perform an analysis of the HSPA + technology, as well as its evolution from the generation of the cellular, in the second stage we will talk about HSPA its advantages, network architecture, structure of protocols in which we will see The MAC layer and the RLC layer, in the third stage HSDPA its network architecture, its main improvements, protocol structure, handover in HSDPA, in the fourth stage HSUPA its network architecture, protocol structure, its main improvements, its channels And handover HSUPA, in stage 5 we will discuss the evolution of HSPA + within release 7

Keywords: HSPA + Technology, MAC, RLC, HSPA, HSDPA, HSUPA, LTE. 


\section{INTRODUCCIÓN}

HSPA evolved es una actualización asequible y gradual para redes HSPA existentes, se proporciona una gran ventaja para los operadores de HSPA, debido a que ofrece un rendimiento impresionante a un costo adicional, algunos operadores HSPA planean utilizar HSPA evolved como compañero a LTE.

Esta Tecnología provee velocidades de hasta 84 Mbps de bajada y 22 Mbps de subida, a través de una técnica multi-antena conocida como MIMO (Multiple-Input Multiple-Output) y modulación 64QAM. Sin embargo, estas velocidades representan picos teóricos que difícilmente se llegan a alcanzar, al lado de la celda (sector, máximo 3 sectores por sitio), se alcanzan velocidades apenas superiores a los 14.4 Mbps de HSDPA, a menos que se utilice un canal mayor a los $5 \mathrm{Mhz}$, las versiones posteriores de HSPA+ soportarán velocidades de hasta 168 Mbps utilizando múltiples portadoras, y hasta $672 \mathrm{Mbps}$ según lo propuesto para la versión 11 de $3 \mathrm{GPP}$, utilizando técnicas avanzadas de antena [1].

\section{GENERACIÓN DE CELULARES}

La evolución de la telefonía celular ha obtenido un gran desarrollo en los últimos años, describiremos a continuación las etapas por la que el celular ha evolucionado[11].

\section{A. Primera Generación}

Tenemos los siguientes estándares AMPS (advanced Mobile Phone System ) fue el sistema analógico basado en FDMA y fue el analógico más usado, NMT (Nordic Mobile Telephone) con sus dos variantes NMT-450 y NMT-900, los números indican las frecuencias utilizadas [11].

\section{B. Segunda Generación}

Los estándares utilizados son D-AMPS que utiliza TDMA, GSM que también utiliza TDMA y CDMA IS-95 basado en tecnología CDMA [2].

\section{Generación $2.5 \mathrm{G}$}

Los estándares que utiliza son GPRS que es una red de conmutación de circuitos ,HSCSD utiliza conmutación de circuitos, EDGE está basada en las especificaciones del Release 98 de 3GPP [3].

\section{Tercera Generación $3 G$}

Sistemas digitales de alta velocidad para voz video datos e internet, esta tecnología es la respuesta a la especificación IMT-200 de la Unión Internacional de telecomunicaciones.

Adicionalmente IMT-2000 ha definido velocidades de transmisión para que una tecnología sea considerada como $3 \mathrm{G}$.

\section{E. Generación 3.5 y $3.75 G$}

Es en esta generación donde aparece la tecnología HSPA, la misma que alcanza velocidades de transmisión de 14.4 Mbps en el enlace descendente y 5.7 Mbps en enlace ascendente, HSPA+ está definida en el raleace 7 de 3GPP la misma que ofrece velocidades máximas de $42 \mathrm{Mbps}$ en el enlace de bajada (downlink) y 22Mbps de subida (uplink), estas tecnologías son muy rápidas en comparación con las versiones anteriores de $3 \mathrm{GPP}$, para la descarga de un archivo de $20 \mathrm{MB}$ se demoraba alrededor de 30 minutos con GPRS, en la actualidad se puede descargar en tal solo 8 segundos utilizando HSPA + .

\section{F. Cuarta Generación $4 \mathrm{G}$}

Esta generación está basada en IP puede alcanzar velocidades hasta de 100 Mbps en el enlace descendente y $50 \mathrm{Mbps}$ en el enlace ascendente, utiliza OFDMA y MIMO.

\section{ACCESO DE PAQUETES DE ALTA VELOCIDAD (HSPA)}

Esta tecnología surge de la combinación de tecnologías posteriores y complementarias a la tercera generación de telefonía móvil 3G UMTS, el objetivo es aumentar la tasa de transferencia de datos a través de un teléfono móvil o dispositivo para laptop o PC, mejorar la calidad de servicio (QoS),la eficiencia del espectro, de esta forma satisfacer la creciente demanda de servicios de datos inalámbricos.

\section{A. Ventajas de HSPA}

Una de las principales ventajas ofrecidas por esta tecnología son las altas velocidades de datos y bajos retardos, puede operar con las mismas frecuencias que opera WCDMA lo que permite reducir costos en el momento de de realizar una migración hacia HSPA, WCDA también puede soportar los servicios de voz y datos simultáneamente sobre la misma portadora, esto es también aprovechado por HSPA lo que permite que el espectro sea utilizados de una manera eficiente.

Arquitectura de red HSPA

En el release 99, la administración de recursos de radio, era controlado por el RNC, no así en el release 
5 y relese 6 , donde el nodo $\mathrm{B}$, controla programación de paquetes en función de la calidad del canal de radio de un UE dato esto disminuye la latencia.

En la figura 1 se puede observar las funcionalidades reasignadas a cada elemento de red según la versión 6 para HSPA, el RNC servidor (SRNC) manejaría la asignación de los canales dedicados (DCHs) manejaría la asignación de los canales, como el canal d envío FACH o el canal descendente compartido DSCH.

El RNC servidor mantiene el control del handover y decide los parámetros de QoS, además el nodo B se encarga de un rápido control de potencia, la programación (scheduling)[11].

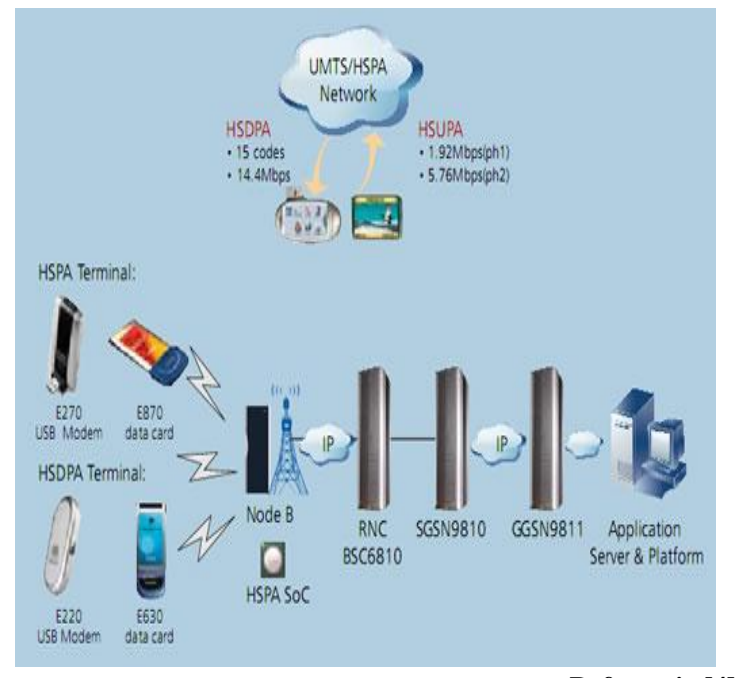

Fig. 1. Arquitectura de red HSPA

Referencia [4]

\section{B. Estructura de Protocolos HSPA}

Las capas de protocolos en el plano de control no cambian con respecto a UMTS, pero en el plano de usuario se modifica tanto en la capa MAC como RLC de acuerdo como se observa en la figura 2.

Capa MAC. Se añaden nuevas subcapas como son MAC-hs para HSPDA, lo cual se encarga principalmente del protocolo $\mathrm{H}$-ARQ en el nodo $\mathrm{B}$, En HSUPA se agrega las subcapas MAC-e y MACes.

Capa RLC. Es esta capa existen mejoras con respecto a UMTS pues se encarga de la retransmisión de paquetes si en las capas inferiores existen errores en la transmisión, después de un numero máximo de retransmisiones o en conexiones móviles [13]

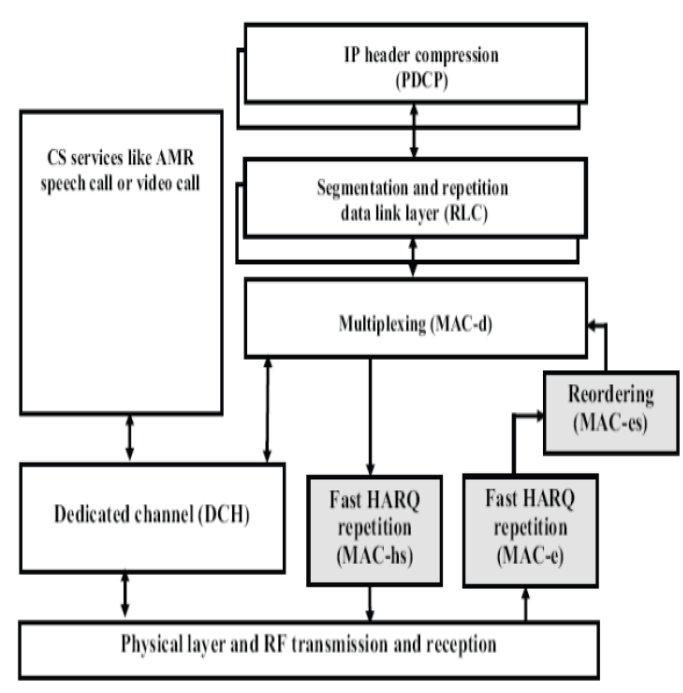

Fuente: HSDPA/HSUPA FOR UMTS

Fig. 2. Estructura de protocolos HSPA para transmisión de datos

\section{ACCESO de PaQUeTES de Alta Velocidad EN EL ENLACE DESCENDENTE (HSDPA).}

Esta tecnología se especifica en el release 5 de 3GPP, agrega un nuevo canal de transporte de datos, el high speed downlink shared channel (HS-DSCH), el cual proporciona un soporte para el alto rendimiento de paquetes de datos en el enlace descendente con respecto al canal dedicado del release 99 .

En el release 99 existen dos canales adicionales para transmitir datos que son el forward access channel y el downlink shared channel (DSCH).

En la siguiente tabla se realiza una comparación entre las características ofrecidas por los canales DCH y HS-DSCH.

La trasmisión HSDPA facilita nuevas características y para soportarlos con un mínimo impacto en la actual arquitectura de protocolo del interfaz de radio, ha sido introducido una nueva subcapa MAC, denominada MAC-hs.

TABLA 1

COMPARACIÓN DE PROPIEDADES FUNDAMENTALES DE DCH Y HS-DSCH

\begin{tabular}{|l|c|c|}
\hline \multicolumn{1}{|c|}{ Características } & DCH (R'99) & HS-DSCH (R'5) \\
\hline Soft Handover & SI & NO \\
\hline $\begin{array}{l}\text { Rápido control de } \\
\text { potencia }\end{array}$ & SI & NO \\
\hline $\begin{array}{l}\text { Codificación y } \\
\text { Modulación } \\
\text { Adaptiva }\end{array}$ & NO & SI \\
\hline $\begin{array}{l}\text { Operación multi- } \\
\text { código }\end{array}$ & SI & SI, EXTENDIDO \\
\hline
\end{tabular}




\begin{tabular}{|l|c|c|}
\hline $\begin{array}{l}\text { Rápido H-ARQ en } \\
\text { capa física }\end{array}$ & NO & SI \\
\hline $\begin{array}{l}\text { Programación por } \\
\text { el nodo B }\end{array}$ & NO & SI \\
\hline
\end{tabular}

\section{A. Arquitectura de red HSPDA}

$\mathrm{La}$ arquitectura se puede observar en la figura 3 , las técnicas básicas HSDPA cuentan con una rápida adaptación a las variaciones de las condiciones del canal de radio.

Uno de los principales objetivos de HSDPA es retener la funcionalidad del release 99 , dividido entre capas y nodos, tanto como sea posible [10].

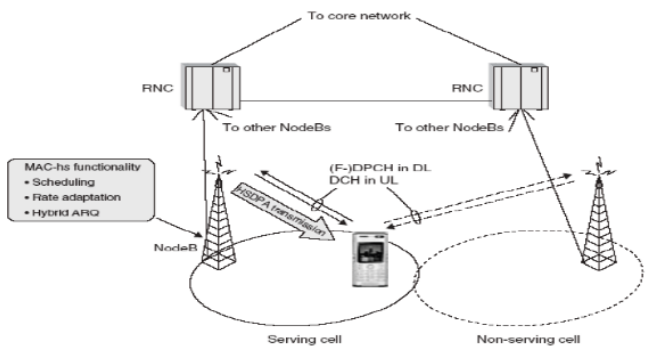

Referencia [5]

Fig. 3. Arquitectura HSDPA

HSPA introduce una nueva subcapa MAC en el nodo B, la MAC-hs, responsable por la programación o despacho, el control de velocidad y operaciones del protocolo H-ARQ, el cual reduce el tiempo de transmisión con respecto a la versión 99 como se observa en la figura 4.

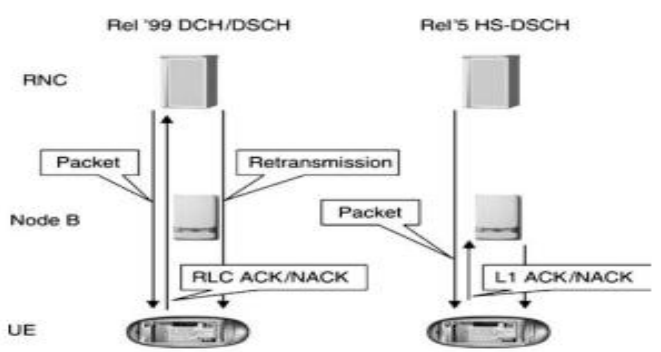

Referencia [5]

Fig. 4. Control de retrasmisión entre el release 99 y release 5

\section{B. Principales mejoras HSPDA}

Las principales mejoras introducidas por esta tecnología son:

- Transmisión en canal compartido.

- Codificación y modulación adaptativa (AMC).

- Petición de retransmisión automática hibrida (H-ARQ)
- Mejora de la planificación o programación del nodo B

- Selección rápida del sitio de la celda (FCSS)

- Pequeño TTI (Trasmission Time Interval).

\section{Estructura de protocolos HSPDA}

Los cambios en la estructura de esta tecnología son evidenciados en la inclusión de la sub-capa MAC-hs, tanto para el UE como para el nodo B, MAC-hs es la encargada de la programación o despacho rápida de canales HS-DSCH en el nodo B, administración de prioridades, selección del formato de transporte y operaciones del protocolo HARQ.

Los datos son enviados en un bloque de transporte hacia la capa física o capa 1, por medio del canal de transporte HS-DSCH, paso su respectivo procesamiento.

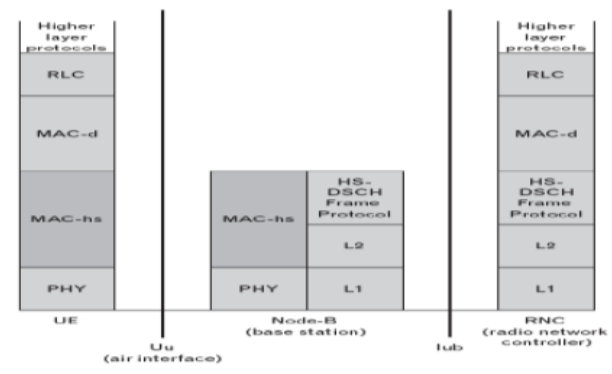

Fig. 5. Estructura de protocolos HSPDA

Referencia [5]

\section{Handover en HSPDA}

Soporta Soft Handover2 en el Uplink. La movilidad, desde celdas que soportan HSDPA a celdas que no soportan HSDPA es manejado fácilmente.

Se puede proveer servicio sin interrupciones a los usuarios, aunque a bajas velocidades de transmisión, usando conmutación de canal compartido en el RNC, y conmutando a dichos usuarios a un canal no dedicado (D-CH en WCDMA) en celdas que no soportan HSDPA.

Similarmente, un usuario equipado con un terminal HSDPA puede conmutar de un canal dedicado a HSDPA, cuando dicho usuario entra a una celda que soporta HSDPA.[10]

\section{ACCESO DE PAQUETES A ALTA VELOCIDAD EN EL ENLACE ASCENDENTE (HSUPA).}

HSUPA (High-Speed Uplink Packet Access) es un protocolo calificado como generación $3.75(3.75 \mathrm{G})$ el cual forma parte de una evolución HSDPA. El protocolo permite tener acceso de datos para redes de 
telefonía móvil con alta tasa de transferencia y está especificado al estándar WCDMA Release 99 (acceso múltiple por división de código de banda ancha basado en estándares de telefonía móvil), tecnología que ofrece una mejora sustancial en la velocidad para el tramo de subida, desde el terminal hacia la red.[10]

La tasa de transferencia indica que teóricamente es hasta $5.76 \mathrm{Mbit} / \mathrm{s}$, aunque en la práctica se hayan comprobados valores menores pero su eficiencia no deja de ser buena. Esta tasa transferencia de datos permite que sea mucho mayor en contraposición a los protocolos UMTS ó EDGE.

Una de las principales utilidades de se dan en usuarios que envían ficheros multimedia directamente a otros, esto incluye imágenes videos en tiempo real a la red o los juegos en tiempo real contra otro jugador, es decir a alta velocidad desde la terminal hacia la red [13].

\section{Arquitectura de Red HSUPA.}

Como HSUPA no constituye en sí mismo una tecnología de acceso, sino más bien una mejora al estándar WCDMA Release 99, del que utiliza una buena parte de sus características básicas para ofrecer un servicio funcional, en la Tabla 2, se muestran ciertas referencias al funcionamiento de la Release 99 para asegurar la comprensión del artículo.

\begin{tabular}{|c|c|l|}
\hline Release & Fecha & Contenido \\
\hline 99 & 2000 Q1 & Primera red UMTS 3G con interfaz aire CDMA \\
\hline R4 & 2001 Q2 & All-IP Core Network \\
\hline R5 & 2002 Q1 & $\begin{array}{l}\text { HSDPA } \\
\text { IMS }\end{array}$ \\
\hline R6 & 2004 Q4 & $\begin{array}{l}\text { HSUPA } \\
\text { MBMS } \\
\text { Diversas mejoras a IMS }\end{array}$ \\
\hline R7 & 2007 Q4 & $\begin{array}{l}\text { Aplicadones real-time (menor latenda, Q0S ...) } \\
\text { HSPA+ } \\
\text { EDGE Evolution }\end{array}$ \\
\hline R8 & 2008 Q4 & $\begin{array}{l}\text { LTE. Nueva interfaz radio OFDMA } \\
\text { Mimo }\end{array}$ \\
\hline R9 & 2009 Q4 & $\begin{array}{l}\text { Interoperabilidad WiMax - LTE/UMTS } \\
\text { Dual-Cell HSDPA con MIMO } \\
\text { Dual-Cell HSUPA }\end{array}$ \\
\hline R10 & - & $\begin{array}{l}\text { LTE Advanced } \\
\text { Multi-Cell HSDPA }\end{array}$ \\
\hline
\end{tabular}

Fig. 6. CONTENIDO DE LAS DISTINTAS RELEASES DE 3GPP

HSUPA, ofrecen altas prestaciones de voz y datos, y permitirá la creación de un gran mercado de servicios IP multimedia móvil, HSUPA mejorará las aplicaciones de datos avanzados persona a persona, con mayores y más simétricos ratios de datos, como el e-mail en el móvil y juegos en tiempo real con otro jugador. Las aplicaciones tradicionales de negocios, junto con muchas aplicaciones de consumidores, se beneficiarán del incremento de la velocidad de conexión [13].

Para incrementar la capacidad y el rendimiento de uplink, HSUPA posee las siguientes características:

\section{A. Reducción del Transmission Time Interval (TTI)}

WCDMA 3GPP Release 99 usa un TTI de $10 \mathrm{~ms}$, $20 \mathrm{~ms}$ o $40 \mathrm{~ms}$ en el uplink. HSUPA opera con un TTI de $2 \mathrm{~ms}$ o $10 \mathrm{~ms}$ en el uplink. Esta reducción del TTI permite una disminución de la latencia total y entrega los medios para que las otras características se adapten rápidamente.

\section{B. Fast hybrid Automatic Repeat reQuest}

El protocolo fast hybrid ARQ usado es similar al de HSDPA. El nodo B rápidamente puede requerir una retransmisión de la información recibida erróneamente, lo que hace al sistema más robusto y una menor latencia. Con HSUPA es posible lograr un mejor Soft handover, para lograrlo todas las estaciones bases y sectores involucrados intentan decodificar la información. Si un ACK (acknowledge) es recibido por al menos uno, el UE considera que la información fue recibida exitosamente.

\section{Fast scheduling}

En el uplink, el recurso común entre los terminales es la cantidad de interferencia tolerable, la cual es la potencia total recibida por la estación base. La cantidad de recursos comunes de uplink que un terminal está usando depende de la tasa de datos usada. Generalmente, mientras mayor sea la tasa de datos, mayor es la potencia de transmisión requerida y mayor es el consumo de recursos. Fast scheduling permite una rápida reubicación de los recursos entre los UEs, también permite al sistema un mayor número de usuarios y adaptaciones rápidas a variaciones de interferencia, lo que lleva a un incremento en la capacidad y probabilidad de que el usuario pueda tener altas tasas de transferencias de datos.

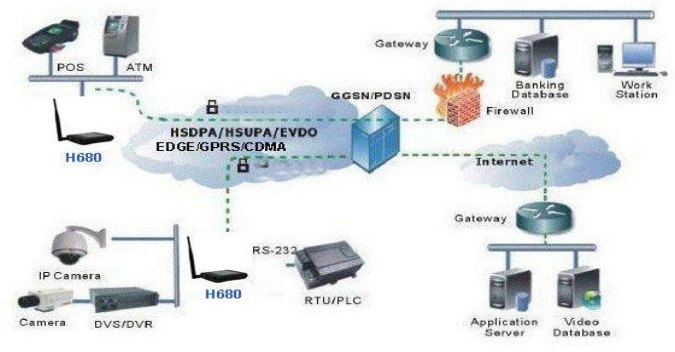

Fig. 7. Arquitectura HSUPA 
El algoritmo de scheduling no está estandarizado y diferentes estrategias pueden ser implementadas. Como muestra la Figura 6. Esta flexibilidad es útil, ya que diferentes ambientes y distintos tipos de tráficos pueden requerir diferentes requerimientos en la estrategia de scheduling. Por ejemplo un UE puede ser provisto desde sólo una o desde varias estaciones bases al mismo tiempo. Así en el último caso, el soft handover debe ser provisto por el uplink mejorado. El UE no sólo es informado sobre el nivel de interferencia en la propia celda, Si no también del nivel de las celdas vecinas. La potencia de salida del UE puede ser reducida si el nivel de interferencia es demasiada alta en las celdas vecinas. También la entrega de soft handover en el uplink provee de una ganancia de $1,5 \mathrm{~dB}$.

En la capa física, HSUPA introduce nuevos canales DE E-AGCH, E-RGCH, F-DPCH, E-HICH, EDPCCH Y E-DPDCH.

E-DPDCH se utiliza para llevar el e-dch canal de transporte, y E-DPCCH se utiliza para transportar la información de control asociada con el E-DCH [13].

\section{PRINCIPALES MEJORAS HSUPA}

Las mejoras que incluye HSUPA son similares a las que ofrece HSDPA las cuales son:

\section{A. Pequeño intervalo de tiempo de transmisión}

UMTS en la version99 emplea un TTI de $10 \mathrm{~ms}$, $20 \mathrm{~ms}$ o $40 \mathrm{~ms}$, en cambio HSUPA emplea un TTI de $2 \mathrm{~ms}$ o $10 \mathrm{~ms}$ en el enlace de subida. Al utilizar un pequeño TTI permite la reducción significativa en la latencia total y proporciona un medio para que las otras mejoras introducidas en HSUPA puedan adaptarse.

\section{B. Rápida transmisión en caso de errores Rápido $H-A R Q$ )}

H-ARQ es un protocolo rápido similar al que usa HSDPA usando la técnica Stop and Wait. La estación base puede solicitar rápidamente una nueva transmisión de datos erróneos recibidos, lo que proporciona mejor robustez y retardos de transmisión bajos.

El softer hanhover es posible para que todas las estaciones o Nodos B involucrados, intenten decodificar los datos recibidos. Si un ACK es recibido de al menos un nodo B, el UE considera que se han recibido satisfactoriamente.

\section{Rápida Propagación}

En enlaces ascendentes el recurso común compartido entre terminales es la cantidad de interferencia tolerable, la cantidad de recursos usados depende de la velocidad de datos usada. Generalmente cuánto más alta sea la tasa de datos, más grande es la potencia de transmisión requerida y más alto el consumo de recursos

La planificación rápida permite la reasignación de recurso rápida entre UEs, explorando las ráfagas en transmisiones de paquetes de datos. Esto también permite al sistema admitir un gran número de usuarios de altas velocidades de datos y rápidamente se adapta a variaciones de interferencia, conduciendo a un aumento en la capacidad como la probabilidad que un usuario experimentará altas velocidades de datos.

Al igual que en HSDPA, HSUPA utiliza un programador de paquetes, pero opera en un principio solicitud de la concesión en los UE piden un permiso para enviar datos y el planificador decide cuándo y cuántos UEs se les permitirá hacerlo. Una petición de transmisión contiene datos sobre el estado de la memoria intermedia de transmisión y la cola en el UE y su margen de potencia disponible. Sin embargo, a diferencia de HSDPA, transmisiones de enlace ascendente no son ortogonales entre sí.

\section{ESTRUCTURA DE PROTOCOLOS HSUPA}

En la estructura se añaden nuevas mejoras en las capas de protocolos de interfaz de radio existentes, HSUPA agrega nuevas entidades en la capa MAC en el plano de usuarios y subcapa MAC-e tanto en losUEs y en los Nodos B. Incluye además la posibilidad de utilizar un TTI 2(Transmission Time Interval- define la longitud de una transmisión en el enlace radio que puede ser decodificada de manera independiente), permitiendo una adaptación más rápida en el enlace descendente, lo que posibilita un mejor aprovechamiento de los recursos disponibles. Todas estas mejoras sobre WCDMA están implementadas dentro de un nuevo canal de transporte denominado E-DC H ( Enhanced Dedicated Channel ).

HSUPA introducen nuevos elementos en la arquitectura. Estas capas funcionales de la MAC pueden operar independientemente de la operaciones de Canales dedicados DCH (Dedicated Channels) de la arquitectura $\mathrm{R}^{\prime} 99$, pero influyen en la limitación de los recursos generales de la interface de aire [13]. 


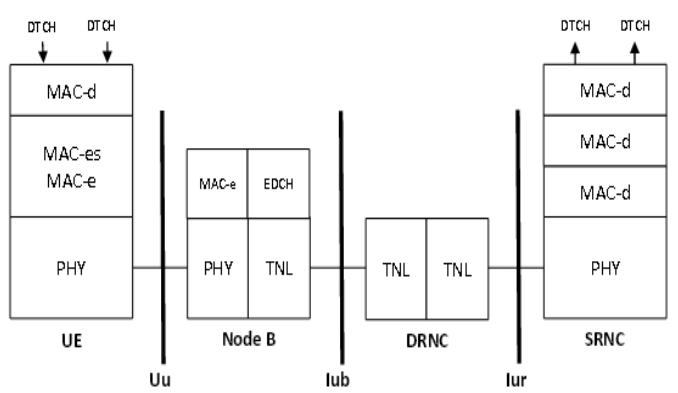

Fig. 8. Estructura del protocolo HSUPA

Para soportar las nuevas capacidades del uplink la arquitectura representada en la Figura7 anterior muestra los nodos modificados con respecto a los utilizados en WCDMA Release 9:

User Equiment: aparece una nueva entidad MACes/MAC-e que será responsable de manejar la retransmisiones HARQ, la asignación de recursos, y la selección TFC del canal E-DCH.

En HSUPA, la implementación de HARQ utilizada es stop-and-wait. Se trata de la forma más sencilla de protocolo, donde el emisor no emite un paquete de información hasta que no haya sido confirmada la correcta recepción del anterior. Básicamente se trata de un protocolo de ventana deslizante con tamaño de ventana 1, por lo que la utilización que se hace del canal es menos eficiente que en otras implementaciones como Go-Back-N ó Selective Repeat.

Nodo B: Su utilidad según la Figura anterior se basa en que un bloque de transporte puede ser recibido en un Nodo B, mientras que otro Nodo B sigue participando en la retransmisión de un bloque de transporte anterior.

S -RNC: aparece la nueva entidad MAC-es para garantizar el correcto orden en la secuencia de datos y permitir la combinación de la información recibida desde distintos Nodos B en caso de soft handover.

Con esta nueva especificación la tasa binaria del uplink puede alcanzar los 5,76 Mbps según la Release 6 del 3GPP. La Release 7 define una nueva categoría 7 que incluye nuevos esquemas de modulación e incrementa la tasa binaria hasta los 11,5 Mbps.

A finales del año 2009, La Release 9 define la categoría 9 que implementa Dual-Cell HSUPA. Esta tecnología, mediante la agregación de recursos en el uplink, permite un mayor aprovechamiento del espectro disponible, llegando a los 23 Mbps. Actualmente no existe ningún despliegue en producción de esta especificación.

HSUPA permite elegir entre dos TTI'de 2 y $10 \mathrm{~ms}$.
El TTI de 2 ms como muestra la Figura 8 minimiza la latencia de transmisión beneficiando a los servicios en tiempo real. El TTI de $10 \mathrm{~ms}$ es necesario por motivos de cobertura para asegurar el servicio en el borde de la célula.

Utilizando un TTI de $2 \mathrm{~ms}$ se obtiene una disminución en el retardo siempre y cuando no sea necesario un número elevado de retransmisiones, y el retardo entre estas sea inferior comparado con el TTI de $10 \mathrm{~ms}$. Sin embargo, a medida que nos aproximamos al borde de la celda, la utilización de esta longitud de intervalo supone un alto consumo de potencia de transmisión. Si además, el número de usuarios activos es elevado, la utilización de un periodo de $2 \mathrm{~ms}$ resulta imposible.

Cuando el terminal se encuentra a grandes distancias de la base, las velocidades de transmisión en el uplink tienen limitaciones como consecuencia de las potencias de transmisión.

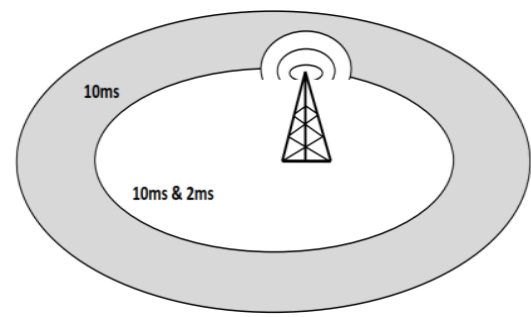

Fig. 9. Transmisión Time Interval (2ms o 10ms)

\section{HANDOVER EN HSUPA}

UMTS soporta 2 clases de handovers y hard handover, pero HSUPA soporta solo soft y soft handover. La principal diferencia entre estos dos es los recursos del sistema involucrados para mantener una llamada o transmisión de los datos.

Un soft handover es hecho antes de la interrupción, por lo cual la comunicación existe entre un UE y más de una celda por cierto período de tiempo. En un hard handover la comunicación se interrumpe antes de ser realizado, por lo cual la comunicación con la primera celda es terminada antes de establecer la comunicación con la segunda celda.

Soft handover: Como muestra la Figura10. Entre 2 celdas o sectores que son soportados por diferentes nodos B. El UE transmite y recibe de ambos nodos B al mismo tiempo, los datos se envían hacia la UE por los 2 nodos B simultáneamente y el UE combina dichos datos. Así mismo el UE envía los datos hacia la RNC, donde se realizan la combinación, en este caso cada nodo B envía órdenes de control de potencia 
al UE

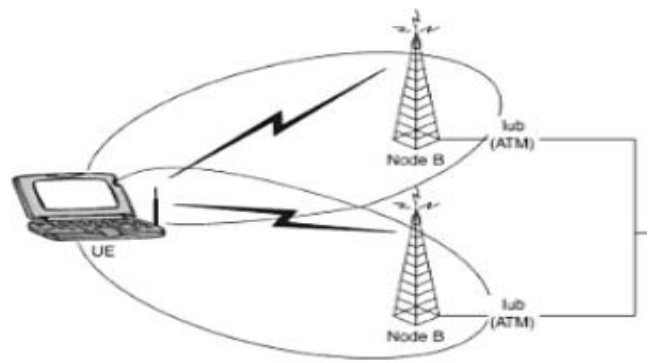

Fig. 10. Soft handover

Softer handover: Ocurre en sectores que son administrados por el mismo nodo B, como muestra la siguiente Figura 10 en este caso solo se activa un lazo de control de potencia y es controlado por el nodo B que sirve a ambas celdas o sectores.

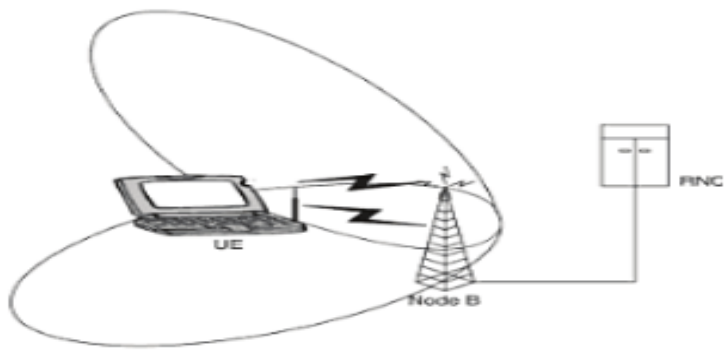

Fig. 11. Softer handover

Dependiendo de la cobertura, un soft y un softer handover pueden ocurrir al mismo tiempo para un UE dado.

\section{EVOLUCIÓN HSPA+ DENTRO DEL RELEASE 7.}

El acceso de paquetes a alta velocidad evolucionado (HSPA+) es un estándar de banda ancha inalámbrico que se define como la versión 7 de 3GPP.

Esta tecnología ofrece velocidades de hasta 42 Mbps en el enlace de bajada y 22 Mbps en el enlace ascendente, las cuales representan velocidades teóricas pico, con tecnologías MIMO (Multiple.Input Multiple-Output), utilizando modulación de orden superior como 64QAM. MIMO en sistemas basados en CDMA actúa como sectores virtuales para dar una mayor capacidad. La velocidad máxima real de un usuario está cerca de los $14 \mathrm{Mbps}$.

HSPA+ también introduce una arquitectura opcional All-IP, para la red donde las estaciones base están directamente conectadas a nodos IP y luego a los routers de frontera que tienen conexión con los ISP's. Además ofrece mejorasen la vida útil de las baterías, una conexión rápida a la red después de un tiempo de inactividad, mayor soporte de servicios en tiempo real como voz sobre IP, juegos en línea y push to talk (PTT) para teléfonos o dispositivos móviles [15].

\section{Estados De Protocolo Con HSDPa Y HSUPA}

Los estados de protocolo de HSDPA y HSUPA son los mismos que los de Release 99. El estado Cell_DCH es usado cuando es posible establecer una comunicación de datos activa hacia y desde el terminal por medio del canal dedicado DCH o HSDPA/HSUPA. En caso de que no halla datos en el buffer, el terminal es movido del estado Cell_DCH al estado ell_FACH o a sus subsiguientes estados, ya sea directamente desde el estado Cell_DCH ó a través del estado Cell_FACH.

Las transmisiones necesitan un tiempo para el proceso de reconfiguración necesitando y manteniendo reservados los recursos del usuario para la transmisión de HSDPA o HSUPA, lo cual no es eficiente para la capacidad del sistema o para el punto de vista del uso de los recursos del BTS. La batería del terminal sufriría un desgaste innecesario al mantener el terminal activo cuando no hay datos pasando por el mismo, causando un rápido agotamiento de la energía de la misma [14].

Los datos pueden ser retransmitidos en el estado Cell_FACH, pero solo usando el canal de acceso directo FACH para la conexión de bajada y el canal de acceso aleatorio RACH en la conexión de subida, lo cual significa que la tasa de transferencia de datos es limitada, por lo que estos canales no ofrecen ninguna de las características de mejora de rendimiento requeridas para HSDPA y HSUPA. Un terminal en el estado Cell_FACH decodifica continuamente el canal FACH para luego iniciar el enlace de bajada de datos (o inicia la transmisión de datos por el buffer de subida) sobre el canal RACH. Dependiendo del volumen de datos (basado en los reportes), el terminal puede volver al estado Cell_DCH.

Si el período de inactividad durante la transmisión de datos continúa por períodos prolongados, se moverá el terminal a los estados Cell_PCH o URA_PCH, estos modos son los que menos potencia consumen y más batería ahorran. El uso de ciclos de recepciones discontinuas mediante la operación de paginado causará algunos retardos adicionales para la continuación de la transmisión de datos, ya que el terminal necesita ser paginado primero [14]. Esto ocurre tanto en una celda o a nivel de área de registración (estado URA_PCH). Este es el beneficio del estado URA_PCH para los terminales con alta

\section{F. Orozco/M. Andrade/J. Orozco/M. Villarroel.}


movilidad, los terminales que se mueven en forma rápida dentro de una red densa, necesitarán muchas actualizaciones de celdas, incrementando la carga de datos del canal RACH, pero necesitarán también ser paginados en múltiples celdas en caso de que exista actividad en el enlace de bajada. Los estados del RRM se muestran en la figura 12 .

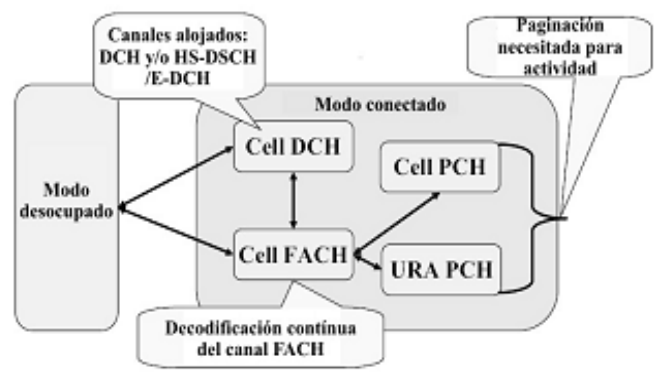

Fig. 12. Estados de RRM

\section{Estado CELL_DCH se caracteriza por:}

* Un canal físico dedicado es alojado en el UE para el enlace de subida y baja.

El UE es conocido a nivel celular de acuerdo a su actual conjunto activo.

\section{Estado CELL_FACH se caracteriza por:}

* Ningún canal dedicado es alojado en el UE.

* El UE monitorea continuamente un canal de acceso directo $(\mathrm{FACH})$ para el enlace de bajada.

* El UE es asignado al canal común por defecto o al canal de transporte compartido para el enlace de bajada (por ejemplo, el $\mathrm{RACH}$ ) que puede ser usado en cualquier momento de acuerdo al procedimiento de acceso para el canal de transporte.

* La posición del UE es conocida por UTRAN a nivel de celda de acuerdo a la celda en la cual el UE realizó la última actualización.

* En el modo TDD, uno o varios canales de transporte USCH o DSCH pueden ser establecidos [15].

\section{Estado CELL_PCH se caracteriza por:}

* Ningún canal físico dedicado es alojado en el UE.

* El UE selecciona un PCH con el algoritmo y usa DRX para el monitoreo del PCH seleccionado por medio del PICH asociado.

* Ninguna actividad de enlace de subida es posible.

* La posición del UE es conocida por UTRAN a nivel de celda de acuerdo a la última actualización realizada por el estado Cell_FACH.

\section{Estado URA_PCH se caracteriza por:}

* Ningún canal físico dedicado es alojado en el UE.

* El UE selecciona un PCH con el algoritmo y usa DRX para el monitoreo del $\mathrm{PCH}$ seleccionado por medio del PICH asociado.

* Ninguna actividad de enlace de subida es posible.

* La posición del UE es conocida por UTRAN a nivel de registración de acuerdo al URA asignado al UE durante la última actualización de URA en el estado Cell_FACH.

\section{SITUACÍON ACTUAL DE HSPA A NIVEL MUNDIAL}

En pocos años el internet a tenido un fuerte impacto en la vida de las personas, convirtiéndose en una necesidad no vital pero si de gran importancia para las actividades diarias, comerciales y profesionales. Para disfrutar de la mayoría de los beneficios de internet el usuario final necesita de una conexión de banda ancha móvil para estar conectados a internet.

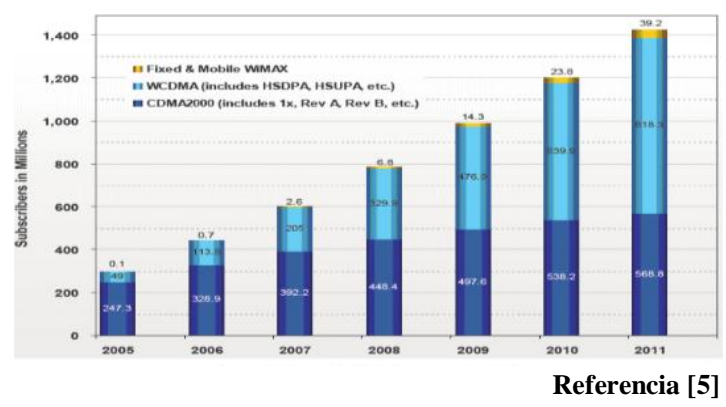

Fig. 13. Suscriptores de banda ancha a nivel mundial

Una serie de tecnologías compiten para ofrecer servicios comerciales de banda ancha móvil, pero la que más éxito ha tenido es HSPA por la adopción y penetración a nivel mundial. Esta tecnología actualmente ha sido implementada por más de 353 operadoras en más de 147 países, y con otros operadores comprometidos a desplegar sus redes adoptando esta tecnología[9]. Según estudios de Qualcomm, la cantidad de suscriptores de banda ancha inalámbrica a nivel mundial en HSPA se esperaba para el 2011 un $57 \%$ de suscriptores a nivel mundial como se muestra en la Figura13, lo cual fue 
corroborado con un pequeño margen de error. Así mismo la suscripción hasta la actual fecha sigue creciendo favorablemente con esta tecnología

Así mismo, del 2012 en adelante la migración a HSPA + evidencia un importante crecimiento según ilustra la figura 14 , preparándose a un cambio en proceso a la tecnología LTE, priorizando al usuario el uso eficiente de voz, datos y video a altas velocidades tanto de subida y bajada.[12]

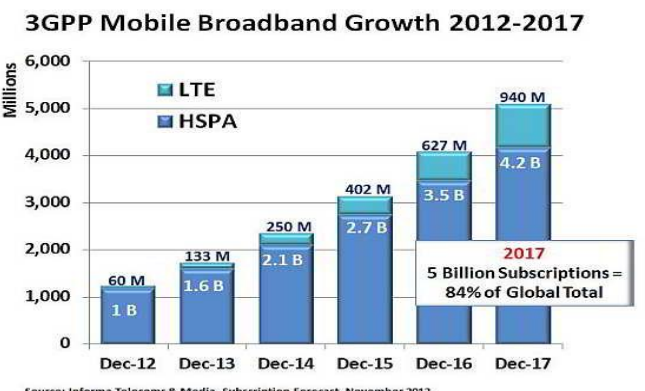

Fig. 14. Pronóstico de crecimiento de suscriptores 3GPP en América (1B = mil millones)

\section{Conclusiones}

Desde el punto de vista de usuario final, quien es el que va a utilizar las bondades de la banda ancha inalámbrica para el acceso al internet resulta mejor el servicio con operadoras que cuenten con HSPA porque estas pueden brindar mayor cobertura, debido al legado obtenido de tecnologías predecesoras como GSM y UMTS del 3GPP

HSUPA no constituye en sí misma un estándar de comunicaciones móviles, sino más bien una mejora al sistema UMTS que en su modalidad más común implementa la tecnología WCDMA en su interfaz radio.

HSUPA es básicamente un canal dedicado, aunque con funcionalidades avanzadas respecto a los canales de dicados de UMTS.

HSDPA soporta aplicaciones que requieren de alta velocidad en una vía tal como el acceso al internet, pero no soporta aplicaciones que requieren de 2 vías como la video conferencia, es por eso que fue desarrollada la tecnología HSUPA para en conjunto con HSDPA formar HSPA, y así brindar altas tasas de datos tanto en enlaces de subida como de bajada

HSPA a HSPA + evolución requiere mucha menos inversión en infraestructura y es menos costosa para mejorar en lugar de LTE que necesita una nueva red construida desde cero.

HSPA + está disponible para cualquier usuario con un teléfono $3 \mathrm{G}$ activado, la evolución a LTE requiere dispositivos destacados de radio LTE específicos y queda mucho por recorrer en cobertura a nivel nacional

Para una nueva entrega de investigación, se analizará la importancia, arquitectura y suscripciones la tecnología LTE

\section{REFERENCIAS}

[1] ITU (Unión Internacional de telecomunicaciones), Generación celular <http://www.itu.int/osg/spu/imt2000/technology.html> [Consulta:13 Sep. 2013]

[2] ITU (Unión Internacional de telecomunicaciones), Generación celular <http://www.itu.int/osg/spu/imt2000/technology.html> [Consulta:16 Sep. 2013]

[3] GPRS \& EDGE , conmutación de circuitos y eficiencia espectral < http://www.3gpp.org/Technologies/KeywordsAcronyms/article/gprs-edge> [Consulta:13 Sep. 2013]

[4] Grupo Banner: Across3G, Arquitectura HSPA release $6<$ http://www.3g.co.uk/PR/Sept2007/5194.htm> [Consulta:12 Sep. 2013]

[5] David Astély, Erik Dahlman, Anders Furuskär, Ylva Jading, Magnus Lindström, and Stefan Parkvall, Abril 2009, Disponible en: http://www.comsoc.org/files/Publications/Tech\%20Focus/pd f/2010/jan-sample/6.pdf .

[6] John Wiley \& Sons Ltd, The Atrium, Southern Gate, Chichester,

West Sussex PO19 8SQ, England, Nov. 2007, $\mathrm{ftp} / / / \mathrm{ftp}$.elet.polimi.it/users/Nicola.Carapellese/Wiley.WCD MA.for.UMTS.HSPA.Evolution.and.LTE.4th.Edition.Nov.2 007.pdf

[7] La inversión extranjera en América Latina, 2007, disponible en: http://www.eclac.org/publicaciones/xml/0/32930/lcg2360e_ Cap_III_f2.pdf [Consulta:13 Sep. 2013]

[8] Serra, J. \& Martínez, V. (2010). Redes de comunicaciones de la telefonía móvil a Internet. Barcelona: Universitat Politècnica de Catalunya

[9] CEPAL. (2008). La inversión extranjera : en América Latina y el Caribe. Santiago: UN/CEPAL

[10] Rábanos, J. (2008). Transmisión por radio. Madrid: Ramón Areces.

[11] Huidobro, J. \& Pastor, R. (2005). Sistemas de telefonía. Madrid: Thomson-Paraninfo

[12] CABREJAS. (2011). 3GPP LTE : hacia la 4g movil. Place of publication not identified: MARCOMBO

[13] Rábanos, J., Tomás, L. \& Salís, J. (2015). Comunicaciones móviles. Madrid: Editorial Universitaria Ramón Areces.

[14] Holma, H., Toskala, A. \& Tapia, P. (2014). HSPA+ Evolution to release 12 : performance and optimization. Chichester, West Sussex, United Kingdom: Wiley.

[15] Sauter, M. (2013). 3g, $4 g$ and beyond bringing networks, devices, and the web together. Chichester, West Sussex, UK: John Wiley and Sons. 